\title{
ON APPLICATION OF ISOGEOMETRIC BEAM ELEMENTS TO MODELING OF GRIDSHELL STRUCTURES
}

\author{
E. Dvořáková* ${ }^{*}$ B. Patzák ${ }^{* *}$
}

\begin{abstract}
Isogeometric analysis has indisputable benefits for the analysis of curved beams. Due to the use of spline basis function (usually NURBS) for both geometry description and unknown approximation, the CAD geometry representation can be directly used for the analysis. Therefore no transformation between CAD and FEM models is needed and the overall design process is more efficient and more accurate. The presented paper concentrates on possibilities of application of IGA to modeling of gridshell structures. The mutual connections of gridshell beams yield in concentrated force and moment loadings, which cause oscillations in numerical solution. This problem is documented on a simple example and the possible solution is proposed.
\end{abstract}

Keywords: $\bar{B}$-method, gridshell structures, isogeometric analysis, numerical locking, Timoshenko curved beam

\section{Introduction}

Isogeometric analysis (IGA), an approach using the same basis functions for both geometry description and unknown approximation, is an alternative to the standard finite element method (FEM) which can be especially profitable for problems of curved geometries. In IGA, the exact geometry representation using NURBS basis functions is directly used for the analysis and unknown approximation, therefore the loss of accuracy caused by geometry approximation is avoided. The model is based on spline basis functions, which allows to describe arbitrarily curved geometries.

In this paper, the focus is on the isogeometric beam element based on application of Timoshenko beam theory, see Zhang et. al (2016). Even though the isogeometric approach can enhance the analysis in the exactness of geometry description, this formulation (as well as standard FEM formulation) still suffers from numerical locking. To overcome the locking phenomena, analogous techniques to standard FEM locking treatment have been studied, see Echter and Bischoff (2010); Bouclier and Elguedj (2012). The presented formulation uses $\bar{B}$-method to overcome the locking phenomena. This method has been chosen for its possibility of use with no prior knowledge of the continuity or degree of the interpolation.

Our goal is to enable the application of the beam element to the analysis of the gridshell structures. These structures are formed from the initially planar grid of mutually connected beams which is subsequently deformed into the desired shape. Possibility of modeling such a structure using isogeometric approach can enhance overall design process as the isogeometric analysis can be easily embedded within design environment thanks to the sharing of the model by both geometry and the analysis. Nevertheless, the isogeometric formulation fails to describe the concentrated moment and force loadings imposed in joints of the individual beams. This paper documents this problem and outlines its possible solution which allows the consecutive development of the interactive design tool for gridshell structures.

\footnotetext{
Ing. Edita Dvořáková: Department of Mechanics, Faculty of Civil Engineering, Czech Technical University in Prague, Thákurova 7; 166 29, Prague; CZ, edita.dvorakova@fsv.cvut.cz

** prof. Dr. Ing. Bořek Patzák: Department of Mechanics, Faculty of Civil Engineering, Czech Technical University in Prague, Thákurova 7; 166 29, Prague; CZ, borek.patzak@fsv.cvut.cz
} 


\section{Isogeometric Timoshenko beam element}

The main difference between standard FEM and IGA is in the type of basis functions which are used for the analysis. While standard finite elements are based on polynomial basis functions, isogeometric approach employs splines. The presented formulation uses NURBS (Non-Uniform B-Splines), as they are probably the most widespread technology in CAD industry.

In IGA, the computational domain is firstly divided into patches, which are further divided into knotspans (seen as elements). Important aspect of NURBS basis functions is that they are generally non-interpolatory and the higher continuity between elements (in so called knots) can be obtained naturally (unlike $C^{0}$ in standard FEM). In the knots, the continuity can be locally decreased up to $C^{0}$ which imposes the interpolatory behavior of the functions at the particular point.

The formulation of the three-dimensional beam element is derived in local coordinate system $(\boldsymbol{t}, \boldsymbol{n}, \boldsymbol{b})$, where $t, n, b$ are tangent, normal and binormal vectors and where the curvilinear coordinate $s$ is measured along the centerline of the beam. There are six independent unknowns: tangential displacement $u_{t}$, normal displacement $u_{n}$, binormal displacement $u_{b}$ and rotations $\theta_{t}, \theta_{n}, \theta_{b}$. A strain-displacement matrix $\boldsymbol{B}$ is defined as

$$
\varepsilon=B r
$$

where $\varepsilon=\left\{\varepsilon_{m}, \gamma_{n}, \gamma_{b}, \chi_{t}, \chi_{n}, \chi_{b}\right\}^{T}$ and $\boldsymbol{r}=\left\{u_{t}, u_{n}, u_{b}, \theta_{t}, \theta_{n}, \theta_{b}\right\}^{T}$. $\boldsymbol{B}$ is derived from the following relations for membrane strain $\varepsilon_{m}$, shear strains $\gamma_{n}$ and $\gamma_{b}$, torsional strain $\chi_{t}$, and bending strains $\chi_{n}$ and $\chi_{b}$

$$
\begin{aligned}
& \varepsilon_{m}=u_{t}^{\prime}-\kappa u_{n}, \quad \gamma_{n}=\kappa u_{t}+u_{n}^{\prime}-\tau u_{b}-\theta_{b}, \quad \gamma_{b}=\tau u_{n}+u_{b}^{\prime}+\theta_{n}, \\
& \chi_{t}=\theta_{t}^{\prime}-\kappa \theta_{n}, \quad \chi_{n}=\kappa \theta_{t}+\theta_{n}^{\prime}-\tau \theta_{b}, \quad \chi_{b}=\tau \theta_{n}+\theta_{b}^{\prime} .
\end{aligned}
$$

Curvature $\kappa$ and torsion $\tau$ are given as

$$
\kappa=\left\|\frac{d^{2} \boldsymbol{r}(s)}{d s^{2}}\right\| \quad \tau=\frac{d \boldsymbol{n}(s)}{d s} \boldsymbol{b}(s),
$$

where $\boldsymbol{r}(s)$ is the position vector. The material stiffness matrix $\boldsymbol{D}$ is given by constitutive relations

$$
\begin{aligned}
& N=E A \varepsilon_{m}, \quad Q_{n}=G A_{n} \gamma_{n}, \quad Q_{b}=G A_{b} \gamma_{b}, \\
& M_{t}=G I_{k} \chi_{b}, \quad M_{n}=E I_{n} \chi_{n}, \quad M_{b}=G I_{b} \chi_{b} \text {. }
\end{aligned}
$$

Finaly, the element stiffness matrix $\boldsymbol{K}$ is defined as

$$
\boldsymbol{K}=\int_{0}^{L} \boldsymbol{B}^{T} \boldsymbol{D} \boldsymbol{B} d s
$$

where the integral is evaluated using Gaussian quadrature, which is not exact for NURBS functions, but it has sufficient accuracy.

\section{Numerical locking}

Due to the same approximation order for displacements and for rotations, the element formulation suffers from numerical locking. In classical FEM, the reduced integration is typically used to overcome this problem, mainly for its computational efficiency. In Isogeometric analysis its use is complicated due to the fact, that the optimal reduced integration rule depends on the choice of approximation order and continuity and such a rule is hard to be determined. On the contrary, Discrete shear gap (DSG) method or $\bar{B}$-method can be used independently on the choice of approximation, the later is used in the presented implementation of the element. 


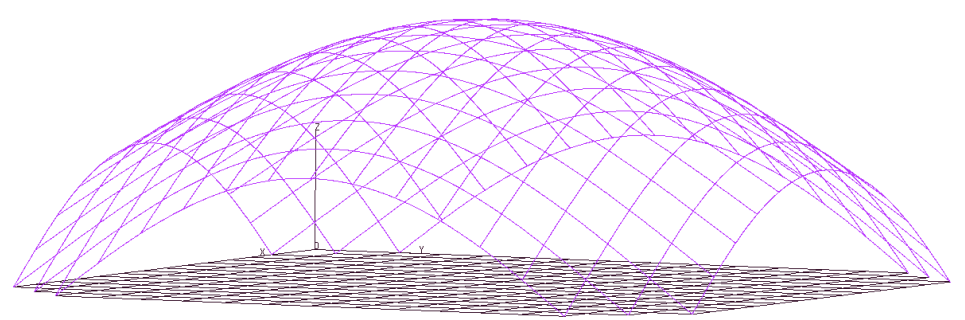

Fig. 1: Gridshell structure modeled and analysed in OOFEM (see Patzák (2018)).

\section{1. $\overline{\mathbf{B}}$ method}

The main idea of the method is to project membrane strain $\varepsilon_{m}$ and shear strains $\gamma_{n}, \gamma_{b}$ onto a basis of lower order. For example, the contribution of membrane strain $\varepsilon_{m}$ to the $\overline{\boldsymbol{B}}$ matrix is

$$
\overline{\boldsymbol{B}}_{m}=\left[\begin{array}{llllll}
\overline{\boldsymbol{B}}_{m}^{1} & \overline{\boldsymbol{B}}_{m}^{2} & \mathbf{0} & \mathbf{0} & \mathbf{0} & \mathbf{0}
\end{array}\right],
$$

where for example

$$
\overline{\boldsymbol{B}}_{m_{i j}}^{1}=\int_{0}^{L} \tilde{N}_{i} N_{j}^{\prime} d s
$$

and where $N_{j}$ is the $j^{\text {th }}$ basis function of the original basis and $\tilde{N}_{i}$ is the $i^{\text {th }}$ basis function of the lower order basis. The membrane contribution to stiffness matrix $\boldsymbol{K}_{m}$ is

$$
\boldsymbol{K}_{m}=E A \overline{\boldsymbol{B}}_{m}^{T} \tilde{\boldsymbol{M}}^{-1} \overline{\boldsymbol{B}}_{m}
$$

where $\tilde{M}$ is the element "mass matrix" calculated in the lower order basis

$$
\tilde{M}_{i j}=\int_{0}^{L} \tilde{N}_{i} \tilde{N}_{j} d s
$$

The analogical procedure can be followed also for the shear strains $\gamma_{n}$ and $\gamma_{b}$.

\section{Application to gridshells}

The design of gridshell structures (see Fig. 1) is complicated process, as the resulting shape of structure depends on initial grid as well as on the history of boundary conditions. In practice, the physical model is often used to determine the shape of the structure and the initial grid at the same time. Introducing Isogeometric analysis into a design of gridshells simplifies the design process as the problem can be easily recalculated when initial grid or boundary conditions change and the resulting shape can be immediately illustrated.

Isogeometric approach offers a possibility to model each grid lath by a single patch. Such a model requires to add additional constraints to enforce the compatibility in joints. To support this approach, the special boundary condition based on Lagrange multipliers method has been implemented. The potential energy is enhanced by an additional term yielding the required continuity

$$
W_{i n t}+\sum_{k} \boldsymbol{\lambda}_{k}\left(\overline{\boldsymbol{r}}_{i}^{k}-\overline{\boldsymbol{r}}_{j}^{k}\right)=W_{\text {ext }},
$$

where $\boldsymbol{\lambda}_{k}$ is vector of Lagrange multipliers for $k^{t h}$ joint, $\overline{\boldsymbol{r}}_{i}^{k}$ and $\overline{\boldsymbol{r}}_{j}^{k}$ are the vectors of constrained unknowns at the $k^{\text {th }}$ joints on the $i^{\text {th }}$ and $j^{\text {th }}$ beams, respectively.

The problem of the presented formulation is that the Lagrange multiplier method results in concentrated force and moment loadings at the joints positions (represented by Lagrange multipliers). The higher continuity of NURBS basis functions along the entire patch results in oscillations in the internal forces, as the basis functions are not able to represent exact solution with discontinuities in internal forces corresponding to concentrated loadings. This problem is illustrated on the example of simply supported beam subjected 

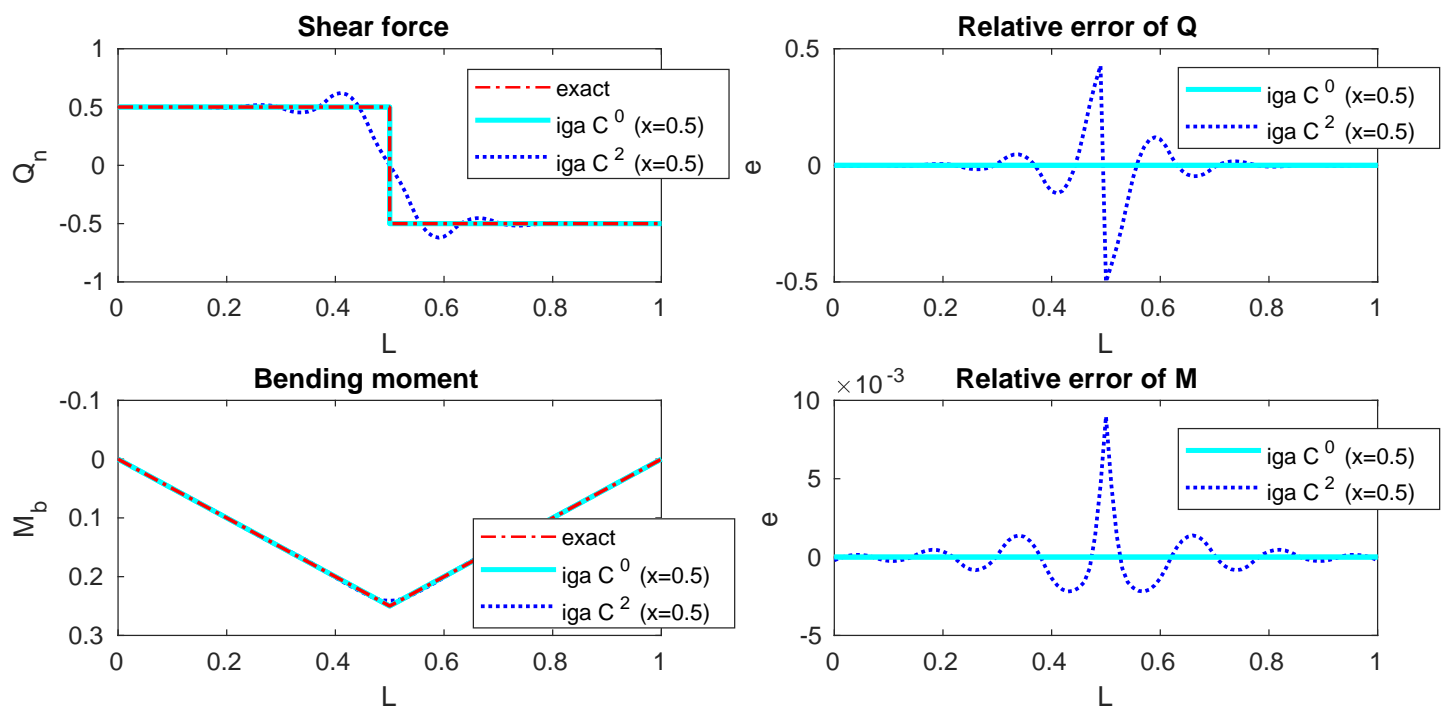

Fig. 2: Example: Simply supported beam subjected to concentrated force in the middle $(L=1, F=1)$. When the force is located at the point with $C^{2}$ continuity, the oscilations in the numerical solution are observed, while for the $C^{0}$ continuity the numerical solution overlaps the exact solution.

to the concentrated force in the middle. For the analysis, thirteen-knotspans patch with the cubic NURBS approximation has been used. The force has been located at the interior knot with $C^{2}$ continuity. This results in oscillations in both shear force and bending moment. On the other hand, when the multiplicity in the particular knot is increased resulting in the $C^{0}$ continuity, the oscillations in the numerical solutions are sufficiently removed. See Fig. 2 for the results. Note, that the increasing knot multiplicity and decreasing the continuity up to $C^{0}$ is analogous to modeling each segment between joints by a single isogeometric element.

\section{Conclusions}

The three-dimensional isogeometric beam element formulation has been presented. The element is based on Timoshenko beam theory and the $\bar{B}$ method is used to remove the numerical locking. The enforcement of continuity of displacements and rotations in joints inevitably leads to application of concentrated forces and moments. Unfortunately, the oscillations in numerical solution of gridshell structure has been documented. It has been shown, that this problem can be solved by a decreasing continuity at the particular location.

Isogeometric analysis can be used to model gridshell structures, but the presented formulation has to be extended for the nonlinear analysis, due to the large deformations. In the future work, our goal is to implement geometrically nonlinear formulation of the element and consequently to develop the interactive design tool for the gridshell structures.

\section{Acknowledgments}

The financial support of this research by the Grant Agency of the Czech Technical University in Prague (SGS project No. SGS18/037/OHK1/1T/11) is gratefully acknowledged.

\section{References}

Bouclier, R., Elguedj, T. (2012), Locking free isogeometric formulations of curved thick beams. Comput. Methods Appl. Mech. Engrg., Vol. 245-246, pp. 144-162.

Echter, R., Bischoff, M. (2010), Numerical efficiency, locking and unlocking of NURBS finite elements. Comput. Methods Appl. Mech. Engrg., Vol. 199, pp. 374-382.

Zhang, G., Alberdi, R., Khandelwal, K. (2016), Analysis of three-dimensional curved beams using isogeometric approach. Engineering Structures, Vol. 117, pp. 560-574.

Patzák, B. (2018), OOFEM project home page, http://www.oofem.org. 